\title{
De una carrera de números primos a una carrera de series divergentes
}

\author{
| From a prime number race to a divergent series race |
}

\section{Gerardo Miramontes de León gmiram@ieee.org \\ Universidad Autónoma de Zacatecas \\ Zacatecas, México}

\author{
Diego Miramontes de León \\ dmiram@uaz.edu.mx \\ Universidad Autónoma de Zacatecas \\ Zacatecas, México
}

Resumen: En la distribución de números primos clases $4 n+3$ y $4 n+1$, se observa una competencia o "carrera" por cuál contiene más números primos. Chébyshev observó que la primera contiene más que la segunda. Aquí se conjetura que hay un infinito número de veces que esta competencia, $\Delta \pi=\pi(4 n+3)-\pi(4 n+1)$, no tiene un líder y que esto ocurre menos veces que la observación de Chébyshev, y más veces que la distribución de Littlewood, es decir, \#\{Infinitas_veces $\Delta \pi>0\}>$ $\#\{$ Infinitas_veces $\Delta \pi=0\}>\#\{$ Infinitas_veces $\Delta \pi<0\}$. Con base en esta idea se presenta una carrera de números subarmónicos divergentes, en la cual la diferencia entre un número subarmónico y otro, es un valor finito que se puede calcular y su valor asintótico es válido para las series infinitas.

Palabras Clave: series divergentes, números primos, infinito

\begin{abstract}
In the distribution of prime numbers classes $4 n+3$ and $4 n+1$, a competition or "race" is observed for which one contains more primes. Chébyshev observed that the former contains more than the latter. Here, it is conjectured that there is an infinite number of times that this competition, $\Delta \pi=$ $\pi(4 n+3)-\pi(4 n+1)$, does not have a leader and that this occurs fewer times than Chébyshev's observation, and more times than Littlewood's distribution, that is, \#\{Infinite_times $\Delta \pi>0\}>\#\{$ Infinite_times $\Delta \pi=$ $0\}>\#\{$ Infinite_times $\Delta \pi<0\}$. Based on this idea, a race of divergent subharmonic numbers is presented, in which the difference between one subharmonic number and another is a finite value that can be calculated and its asymptotic value is valid for infinite series.
\end{abstract}

Keywords: infinite series, prime numbers, infinity

\section{Introducción}

En el estudio de la distribución de números primos, de los cuales hay en un número infinito, hay trabajos muy importantes; comenzando con los teoremas de Euclides, Euler, Fermat, Mersenne, Gauss, Dirichlet, Chébyshev, y muchos más, se puede llegar hasta los días actuales.

Al conocer que hay un número infinito de números primos, interesa relacionar, por cierta afinidad, el tema de progresiones aritméticas infinitas con las series infinitas divergentes. Así pues, partiendo de 
resultados presentados por otros autores [4] con la "carrera de números primos", primero se revisan algunos de esos resultados y después se presenta la "carrera de series divergentes".

En otro artículo se mostró que dos series divergentes tienen diferente valor al infinito [7], es decir, se pueden ver como dos series que llevan una diferencia en su desarrollo y difieren en su valor al infinito. Esta idea de que una cantidad infinita es menor que otra parece respaldada por los teoremas y conjeturas de autores como los mencionados anteriormente. Por ejemplo con Chébyshev, donde se encuentra que algunas secuencias de términos infinitos tienen un número mayor de términos unas de otras.

Una de esas ideas que llevan cuenta de las diferencias entre el número de términos en secuencias o progresiones infinitas es la carrera de números primos. En ese caso lo que importa es la diferencia en el número de términos entre dos secuencias o progresiones aritméticas, mientras que en nuestro caso la diferencia se refiere a la suma de términos, es decir, a la serie.

Como marco de referencia se presentan los resultados de la carrera de los números primos y se destaca que, a diferencia de las series divergentes, el número de veces en que una progresión es mayor que la otra cambia en ciertos intervalos de $n$. En nuestro caso lo que se verifica es que la carrera siempre lleva un líder, pero se determina el valor de la diferencia final, cuando $n$ tiende al infinito.

\section{Objetivos}

El principal objetivo de este trabajo es comprobar de forma numérica lo que se planteó anteriormente en [7] y reforzarlo con los resultados de la carrera de números primos donde se puede asegurar que "un infinito va detrás de otro". Para llegar al objetivo principal, se revisa primero la distribución de números primos, como marco de referencia. Posteriormente, se desarrollan series subarmónicas parciales divergentes de [7], así como las sumas parciales de las series-p convergentes. Al sumar ambas partes se obtiene el número armónico $\mathrm{H}_{n}$. Una vez calculados estos números que llamamos subarmónicos- $p$, se muestra que mantienen una diferencia en su valor con un valor asintótico que tiende a ser igual a la diferencia de sus series-p correspondientes.

\section{Sucesiones de números primos}

\section{Definición 1 (Número primo)}

Se dice que un número natural $p \in \mathbb{N}$ es un número primo, si $p>1$ y tiene solamente dos divisores positivos 1 y $p$.

Los números que no son primos se llaman números compuestos. La importancia de los números primos está en que todo número entero puede expresarse como el producto de dos o más números primos. A esto se le conoce como el teorema fundamental de la aritmética. Al mismo tiempo se tiene la Conjetura de Goldbach: todo número natural mayor que 2 puede expresarse como la suma de tres números primos.

De igual manera, se conoce el siguiente teorema, dado por Euclides en el libro IX de sus Elementos, proposición 13:

\section{Teorema 1 (Euclides)}

Existe un número infinito de números primos. 
La demostración del Teorema 1 se encuentra en casi cualquier texto sobre teoría de números o en la internet, y por lo tanto la omitimos aquí.

Sabiendo que hay un número infinito de números primos, una pregunta inmediata es ¿cuántos números primos hay desde 1 hasta $\mathrm{N}$, donde $\mathrm{N}$ es un número natural? Si consideramos los números del 1 al 10, encontramos que el 2, 3, 5, 7 son números primos, es decir hay 4 números primos.

Encontrar números primos, si el valor de $\mathrm{N}$ es muy grande, se vuelve un problema mayor, especialmente porque no se conoce una fórmula para encontrar el n-ésimo número primo y lo que se han desarrollado son expresiones aproximadas cuando el valor de $\mathrm{N}$ es grande.

La cantidad de números primos menores que una $\mathrm{N}$ dada, se denota como $\pi(\mathrm{N})$.

Definición 2 (Función contadora de primos)

La función contadora de números primos es la función $\pi(\mathrm{N})$ definida como

$\pi(N):=\#\{p \in \mathbb{N} \mid p$ es un número primo y $p \leqslant N\}$ y donde $\#\{\}$ denota su cardinalidad.

Por ejemplo, usando la función primes $(\mathrm{n})$ en GNU Octave [2], los números primos para $n=20$ son: $2,3,5,7,11,13,17,19$. Es decir, $\pi(20)=8$.

\section{Teorema 2 (Dirichlet)}

Si $\operatorname{mcd}(q, a)=1$, entonces la progresión aritmética $\{q n+a\}_{n \in \mathbb{N}}$ contiene infinitos números primos, donde $\operatorname{mcd}(q, a)$ denota al máximo común divisor de $q$ y a, y siempre que q y a sean coprimos.

Entonces, como consecuencia del Teorema 2 , surge el problema de la distribución de números primos según diferentes clases de residuos. Por ejemplo, tomando el número 4 es posible repartir los números naturales en grupos distintos: los números que son múltiplos de 4 , los que son de la forma $4 \mathrm{n}+3$ y los que son de la forma $4 n+1$, llamados clase residual 3 módulo 4 , a los primeros, y clase residual 1 módulo 4, a los segundos. En general, se tiene que dado un número q, las únicas clases residuales módulo q que pueden contener infinitos números primos son las de la forma $q n+a$, donde a es un número entre 1 y q -1 , que además es coprimo con q. De este modo si q es 4 la única selección posible de a es 1 y 3 . Los valores posibles de a se denotan por $\phi(n)$ y se llaman clases residuales donde todos los números primos se distribuyen. A cada uno de estos conjuntos se le llama clase primitiva de residuos.

Nuestro interés se centra en los números primos impares, divididos en dos clases, dependiendo del resto que resulta al dividirlos entre 4. En particular éstas son las clases módulo 4; una clase está formada por los primos de la forma $4 n+3$, y la otra clase por los primos de la forma $4 n+1$, es decir, aquellos que tienen un residuo 3 y un residuo 1, respectivamente. Al contar la cantidad de números primos en cada clase hasta un número dado $n$, se encuentra que hay una desproporción entre ellos.

Este fenómeno fue observado por primera vez por Chébyshev [3],

Hay una notable diferencia cuando dividimos los números primos en las dos formas $4 n+3$ y $4 n+1$ :

la primera de ellas contiene una cantidad mayor que la segunda.

Desde entonces se ha tratado de comprobar dicha diferencia, llegando a decirse que la observación de Chébyshev se presenta infinitas veces.

La cantidad de números primos de la forma $4 n+3$ y $4 n+1$, también se denotan por $\pi(n ; 4,3)$ y $\pi(n ; 4,1)$, hasta un número $n$ deseado. 
Definición 3 (Función contadora de primos módulo q)

La función contadora de números primos con residuo a módulo q queda designada por: $\pi(n ; q, a):=\#\{p \in \mathbb{N} \mid p$ es primo, $p \leqslant n: p \equiv a(\bmod q)\}$.

Sea

$$
\Delta \pi(n)_{4,3,1}=\pi(n ; 4,3)-\pi(n ; 4,1)
$$

la diferencia entre el número (o cantidad) de números primos de la clase $4 n+3$ y $4 n+1$. Rubinstein y Sarnak demostraron en 1994 que en el $99.6 \%$ de los casos $\pi(n ; 4,3)$ es mayor que $\pi(n ; 4,1)$ [8]. Esto confirma, al no ser el $100 \%$, que en algunas raras ocasiones el signo de la diferencia (1) se vuelve negativo. Pero como hay infinitos números primos, las pocas veces que la diferencia es negativa también ocurre infinitas veces. De ahí se obtiene la conclusión que el infinito número de veces que la diferencia es negativa va atrás del infinito número de veces que la diferencia es positiva, en otras palabras, un infinito es menor que el otro.

\section{Revisión de algunos resultados publicados por otros autores}

Se puede ver que la distribución de números primos en clases primitivas distintas, para el módulo 4 , está en realidad igualmente dividida. Utilizando Octave, se calculó $\pi(n ; 4,3)$ y $\pi(n ; 4,1)$, mostrados en la Tabla 1, donde se puede ver que son coincidentes con los resultados publicados en [4], pero hemos incluido una cuarta columna, la cual será útil en la siguiente sección, para mostrar el comportamiento del valor de $\Delta \pi(n)_{4,3,1}$, es decir, la diferencia entre ambas clases de números primos.

Tabla 1: Carrera de números primos.

\begin{tabular}{cccc}
\hline $\mathrm{n}$ & $\pi(\mathrm{n} ; 4,3)$ & $\pi(\mathrm{n} ; 4,1)$ & $\Delta \pi(\mathrm{n})_{4,3,1}$ \\
\hline 100 & 13 & 11 & 2 \\
200 & 24 & 21 & 3 \\
300 & 32 & 29 & 3 \\
400 & 40 & 37 & 3 \\
500 & 50 & 44 & 6 \\
600 & 57 & 51 & 6 \\
700 & 65 & 59 & 6 \\
800 & 71 & 67 & 4 \\
900 & 79 & 74 & 5 \\
1000 & 87 & 80 & 7 \\
2000 & 155 & 147 & 8 \\
3000 & 218 & 211 & 7 \\
4000 & 280 & 269 & 11 \\
5000 & 339 & 329 & 10 \\
6000 & 399 & 383 & 16 \\
7000 & 457 & 442 & 15 \\
8000 & 507 & 499 & 8 \\
9000 & 562 & 554 & 8 \\
10000 & 619 & 609 & 10 \\
20000 & 1136 & 1125 & 11 \\
50000 & 2583 & 2549 & 34 \\
100000 & 4808 & 4783 & 25 \\
\hline
\end{tabular}


Dados los casos de la Tabla 1, y de acuerdo al Teorema 1, se puede proponer, que

$$
\#\left\{\Delta \pi(n)_{4,3,1}>0\right\} \rightarrow \infty
$$

es decir, hay un número infinito de veces en que $\pi(n ; 4,3)>\pi(n ; 4,1)$.

Pero al mismo tiempo, el teorema de Dirichlet, conocido como el teorema de números primos para progresiones aritméticas, indica que para cualquier módulo q los números primos tienden a estar igualmente distribuidos entre las varias formas $q n+a$ donde el máximo común divisor entre $q$ y a es 1. Esto necesariamente nos llevaría a asegurar que

$$
\frac{\pi(n ; 4,3)}{\pi(n ; 4,1)} \rightarrow 1
$$

lo cual, de cumplirse, nos presenta la pregunta si la (3) contradice la (2). Lo interesante es que, como se muestra más adelante, una no excluye a la otra. En la Tabla 2 se observa el comportamiento de la (3) para unos pocos valores de $n$. Para los casos en que $n$ va de $10^{1}$ a $10^{9}$ se observa que (3) tiende a 1 .

Tabla 2: Relación entre dos clases según Dirichlet y ecuación (3).

\begin{tabular}{crrr}
\hline $\mathrm{n}$ & $\pi(\mathrm{n} ; 4,3)$ & $\pi(\mathrm{n} ; 4,1)$ & $\pi(\mathrm{n} ; 4,3) / \pi(\mathrm{n} ; 4,1)$ \\
\hline $10^{1}$ & 2 & 1 & 2.00000000000000 \\
$10^{2}$ & 13 & 11 & 1.18181818181818 \\
$10^{3}$ & 87 & 80 & 1.08750000000000 \\
$10^{4}$ & 619 & 609 & 1.01642036124795 \\
$10^{5}$ & 4808 & 4783 & 1.00522684507631 \\
$10^{6}$ & 39322 & 39175 & 1.00375239310785 \\
$10^{7}$ & 332398 & 332180 & 1.00065627069661 \\
$10^{8}$ & 2880950 & 2880504 & 1.00015483401516 \\
$10^{9}$ & 25424042 & 25423491 & 1.00002167286939 \\
\hline
\end{tabular}

Hay que notar que la (2) indica solamente que el número de veces que $\pi(n ; 4,3)>\pi(n ; 4,1)$ es infinito, pero no dice en qué medida es mayor. Como se podrá comprobar, hay valores de $n$ en los cuales la diferencia es mínima, es decir $\pi(n ; 4,3)-\pi(n ; 4,1)=1$. Más aún, hay también casos en que la diferencia es cero, pero eso no se ha tomado en cuenta, aunque implícitamente está en la (3).

Para hacer más interesante el problema, resulta que aunque el número de veces que (1) es negativa es pequeño, al contar con infinitos números primos, este caso también debe ser infinito. $\mathrm{Al}$ respecto, en 1914, E. Littlewood presentó el siguiente teorema [6]:

\section{Teorema 3 (J. E. Littlewood)}

Hay valores arbitrariamente grandes de $x$ para los cuales hay más primos de la forma $4 \mathfrak{n}+1$ hasta $x$ que primos de la forma $4 n+3$. De hecho, hay valores arbitrariamente grandes de $x$ para los cuales

$$
\{\pi(n ; 4,1) \leqslant x\}-\{\pi(n ; 4,3) \leqslant x\} \geqslant \frac{1}{2} \frac{\sqrt{x}}{\ln x} \ln (\ln (\ln x))
$$

En el Teorema 3, al ser citado por [4], se emplea el término "valores arbitrariamente grandes", es decir, no indica todavía que $n$ tienda a infinito, como se hace a continuación.

A partir de la (4) se refuerza nuestra observación anterior, es decir, que cuando $n$ tiende a infinito, el número de veces que (1) es negativa, también tiende a infinito, aunque muy lentamente.

La historia no termina aquí, pues Knapowski y Turán hicieron una conjetura que sigue la observación de Chébyshev [5]: 


\section{Conjetura 1 (Knapowski, Turán)}

En tanto $X \rightarrow \infty$ el porcentaje de enteros $x \leqslant X$ para el cual hay más primos de la forma $4 n+3$ hasta $x$ que de la forma $4 n+1$ tiende al $100 \%$.

La Conjetura 1 no se cumple, como se aclara más adelante.

\section{Calculando $\pi(n)$ para diferentes clases primitivas}

Los resultados mostrados en la Tabla 1 deben ser tomados con precaución, ya que si observamos todos los valores de $n$, de uno en uno, desde 100 hasta 100 mil, entonces sí aparecen valores en los que (1) es negativa. Aunque de los cerca de 100 mil valores de $n$ sólo aparece un número pequeño de veces con signo negativo, hay un número mucho mayor en que $\Delta \pi(n)_{4,3,1}$ es cero. Contar el número de veces en que $\Delta \pi(n)_{4,3,1}=0$ no ha llamado mucho la atención hasta ahora, sin embargo, puede ser una cantidad importante ya que, para ciertos rangos de $n$, es incluso varias veces mayor al número en que hay un cambio de signo a negativo.

En [4], página 3, se presenta una tabla indicando el "Máximo porcentaje de $x \leqslant X$ para el que hay más primos $4 n+1 \leqslant x$ que $4 n+3$ ". En la primera línea aparece $x$ en el rango $0-26860$ y el porcentaje es $0 \%$, lo cual es verdad, pero no se menciona que en ese rango de $x$ hay 29 veces (28 si partimos de 1 a 26860 ) en que $\Delta \pi(n)_{4,3,1}=0$, por lo tanto el porcentaje en que $\Delta \pi(n)_{4,3,1}>0$ no es el $100 \%$.

Un examen detallado, en este pequeño intervalo de $n$, nos muestra los siguientes resultados: En todo el intervalo no ocurre ningún cambio de signo en $\Delta \pi(n)_{4,3,1}$, sin embargo sí hay 28 veces en que es igual a cero, es decir, $\pi(n ; 4,3)=\pi(n ; 4,1)$. Por otro lado, la diferencia mínima entre $\pi(n ; 4,3)$ y $\pi(n ; 4,1)$ es 1 , y eso ocurre 122 veces, mientras que el valor máximo es 31, el cual ocurre solamente 6 veces.

Si no se toma en cuenta el número de veces en que $\pi(n ; 4,3)=\pi(n ; 4,1)$, entonces diríamos que, en este pequeño intervalo, se cumple la Conjetura 1. Lo mismo ocurre si sólo se toman los resultados mostrados en la Tabla 1. Por lo anterior se calculó, en un segundo ejercicio, la carrera entre estas dos clases de primos tomando todos los valores de $n$ desde 1 hasta 100 mil.

Para dar una idea de los intervalos en que la carrera cambia de líder, se incluyen los siguientes intervalos para el caso 1 a $100 \mathrm{mil}$ :

- Se encuentran valores negativos de $\Delta \pi(n)_{4,3,1}$ en $n=26861$ y 26862.

- Se encuentran algunos valores cero en $n=1,2,5,6,17,18,41,42,461$ y 462 , después para los intervalos $n=$ [26833:26838], [26849:26860], [26863:26878], y finalmente en $n=26881$.

Otros datos que se observan para $\Delta \pi(n)_{4,3,1}$ en este intervalo ( 1 a $\left.100 \mathrm{mil}\right)$ son:

- Valor mínimo positivo $=1$, ocurre 164 veces. Valor máximo positivo $=47$, ocurre 18 veces.

- Valor mínimo negativo = Valor máximo negativo $=-1$, ocurre 2 veces.

- Valor $=0$, ocurre 70 veces.

Este tipo de detalles se obtuvieron en otros intervalos. Para $n$ de 1 a 500 mil se observa lo siguiente:

- Valor mínimo positivo $=1$, ocurre 164 veces. Valor máximo positivo $=105$, ocurre 26 veces.

- Valor mínimo negativo = Valor máximo negativo = -1, ocurre 2 veces. 
- Valor $=0$, ocurre 70 veces.

Para $n$ de 1 a 1 millón se observa lo siguiente:

- Valor mínimo positivo $=1$, ocurre 1524 veces. Valor máximo positivo $=157$, ocurre 36 veces.

- Valor mínimo negativo $=-8$, ocurre 2 veces. Valor máximo negativo $=-1$, ocurre 2 veces.

- Valor $=0$, ocurre 1532 veces.

En este punto conviene hacer una comparación visual del comportamiento de la carrera $\pi(n ; 4,3)$ contra $\pi(n ; 4,1)$, la cual se muestra, para $n$ de 1 a 100 mil, en la Figura 1.

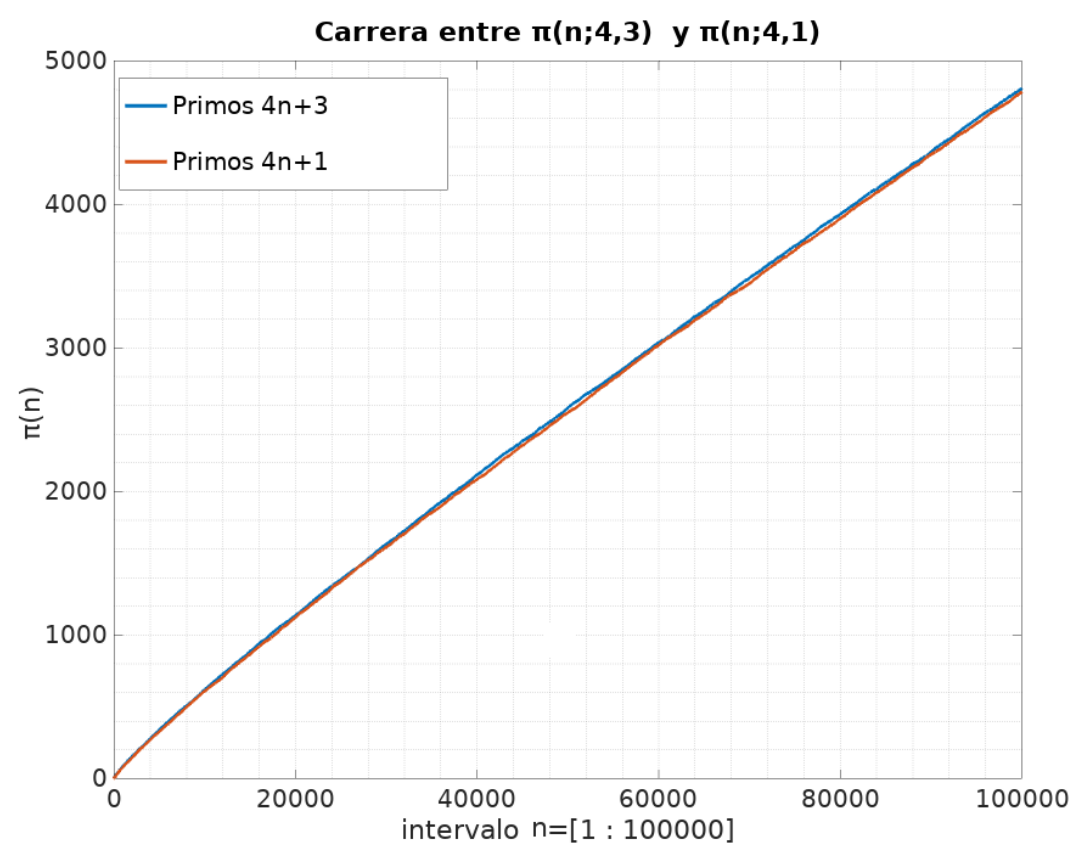

Figura 1: Carrera entre números primos de 1 a 100 mil. (Elaboración propia).

Ambas clases de números primos contienen un número muy similar de términos, según muestra la proximidad de ambos trazos.

Comparando los datos reportados por Andrew Granville y Greg Martin [4] donde, para el intervalo de 0 a $10^{5}$, indican que sólo el $0.01 \%$ ocurren valores negativos, aquí reportamos que en realidad ese porcentaje corresponde a $0.0004 \%$ para los valores negativos y $0.0124 \%$ para valores iguales a cero. Sumando ambos porcentajes, efectivamente se tiene $0.0128 \%$. En otros intervalos esos porcentajes cambiarán, pero quizá se deba hacer la distinción entre ambos casos, es decir, el porcentaje para $\Delta \pi(n)_{4,3,1}>0$, el porcentaje para $\Delta \pi(n)_{4,3,1}<0$ y el porcentaje para $\Delta \pi(n)_{4,3,1}=0$. Por supuesto que se necesitan muchos más cálculos, para valores de $n$ realmente grandes.

Sería impráctico mostrar todos los valores en los que (1) es negativa y cero, así que en la Tabla 3 se muestra un resumen para diferentes intervalos de $n$. En la primera columna se muestra el intervalo de $n$, en la segunda columna $\Delta \pi(n)_{4,3,1}$, en la tercera columna se indica el número de veces en que $\Delta \pi(n)_{4,3,1}$ es mayor que cero, menor que cero y cero. En la cuarta columna se muestran los porcentajes para cada caso.

Finalmente, ya que se ha hecho la conjetura de que al haber infinitos números primos, las pocas veces que $\Delta \pi(\mathrm{n})_{4,3,1}<0$ también debe ser infinito, hacemos la siguiente conjetura: 
Tabla 3: Valores positivos, negativos y cero para $\Delta \pi(n)_{4,3,1}$ en el intervalo $n=[a: b]$.

\begin{tabular}{|l|l|l|l|}
\hline $\mathrm{n}=[\mathrm{a}: \mathrm{b}]$ & & & \\
& $\Delta \pi(\mathrm{n})_{4,3,1}$ & \# eventos & $\%$ \\
\hline \hline$\left[1: 1 \times 10^{5}\right]$ & $\Delta \pi(\mathrm{n})_{4,3,1}>0$ & 99928 & 99.928 \\
& $\Delta \pi(\mathrm{n})_{4,3,1}<0$ & 2 & 0.0020 \\
& $\Delta \pi(\mathrm{n})_{4,3,1}=0$ & 70 & 0.070 \\
& Total & 100000 & 100 \\
\hline$\left[1: 5 \times 10^{5}\right]$ & $\Delta \pi(\mathrm{n})_{4,3,1}>0$ & 499928 & 99.9856 \\
& $\Delta \pi(\mathrm{n})_{4,3,1}<0$ & 2 & 0.0004 \\
& $\Delta \pi(\mathrm{n})_{4,3,1}=0$ & 70 & 0.014 \\
& Total & 500000 & 100 \\
\hline$\left[1: 1 \times 10^{6}\right]$ & $\Delta \pi(\mathrm{n})_{4,3,1}>0$ & 995242 & 99.5242 \\
& $\Delta \pi(\mathrm{n})_{4,3,1}<0$ & 3406 & 0.3406 \\
& $\Delta \pi(\mathrm{n})_{4,3,1}=0$ & 1352 & 0.1352 \\
& Total & 1000000 & 100 \\
\hline
\end{tabular}

\section{Conjetura 2 (Este trabajo)}

Para $n \rightarrow \infty, \#\left\{\Delta \pi(n)_{4,3,1}=0\right\} \rightarrow \infty$, es decir, el número de veces en que el número de primos de la forma $4 n+3$ es igual al número de primos de la forma $4 n+1$, es infinito.

Una base para esta conjetura son los Teoremas 1 y 2 . Así como se cumple (2), porque hay un número infinito de números primos, por la misma razón se cumple la (3). Lo que sí se puede asegurar es que la Conjetura 1 no se cumple, ya que al expresarla al $100 \%$ excluye necesariamente a una de las dos.

\subsection{En busca de valores negativos y cero}

Para respaldar la Conjetura 2, se cambia el enfoque de calcular uno a uno los números primos para valores muy grandes de $n$. Tomando 5 mil números al azar, con un valor máximo de $n=10^{9}$ se construyó la Figura 2a. En los datos numéricos se encontró un valor $\Delta \pi(n)_{4,3,1}=0$ en $n=951890755$. Esto indica que alrededor de este valor de $n$ habrá una región en la cual las dos clases de números primos se aproximan (de hecho para ese valor de $n$ son iguales) y que puede haber cambio de signo.

La Figura 2b muestra el equivalente a una ampliación de la Figura 2a.

Enseguida se procedió a calcular $\Delta \pi(n)_{4,3,1}$ en un intervalo $n=$ [951890001:951891499], es decir en 1500 puntos alrededor. Visualmente la Figura 2a no permite leer si hay valores negativos, ya que por lo regular el número de veces que $\Delta \pi(n)_{4,3,1}>0$ es mayor que el número de veces que $\Delta \pi(n)_{4,3,1}<0$, de modo que se tienen que leer a los datos numéricos. En la Tabla 4 se muestra un resumen de resultados. Puede notarse que el procentaje de eventos en cero es mayor que el de eventos negativos.

Tabla 4: $\Delta \pi(\mathrm{n})_{4,3,1}$, en el intervalo $\mathrm{n}=[951890001: 951891499]$.

\begin{tabular}{llc}
\hline$\Delta \pi(n)_{4,3,1}$ & \# eventos & $\%$ \\
\hline$\Delta \pi(n)_{4,3,1}>0$ & 915 & 60.959 \\
$\Delta \pi(n)_{4,3,1}<0$ & 258 & 17.189 \\
$\Delta \pi(n)_{4,3,1}=0$ & 328 & 21.852 \\
\hline Total & 1501 & 100 \\
\hline
\end{tabular}




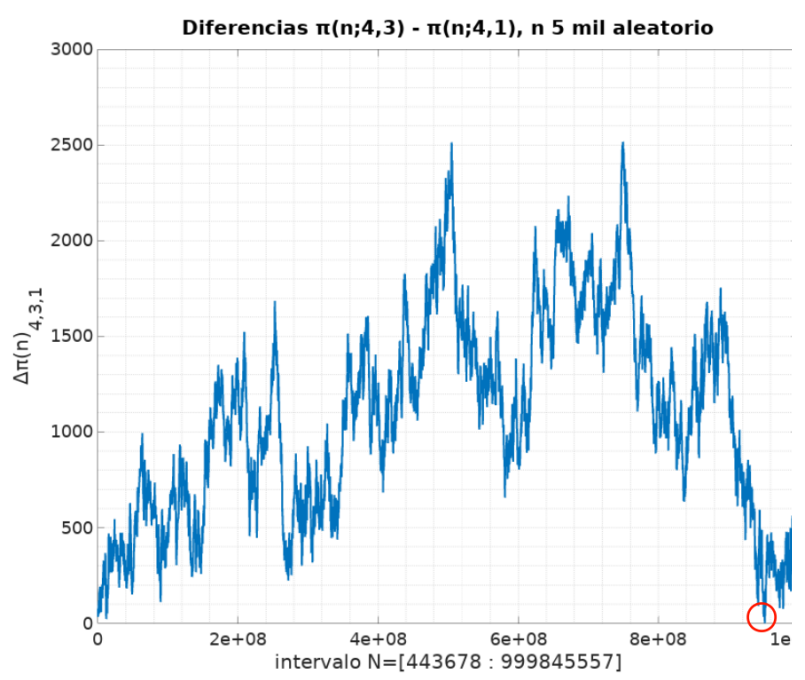

(a) Diferencia $\Delta \pi(n)_{4,3,1}$ con $n \approx 10^{9}$

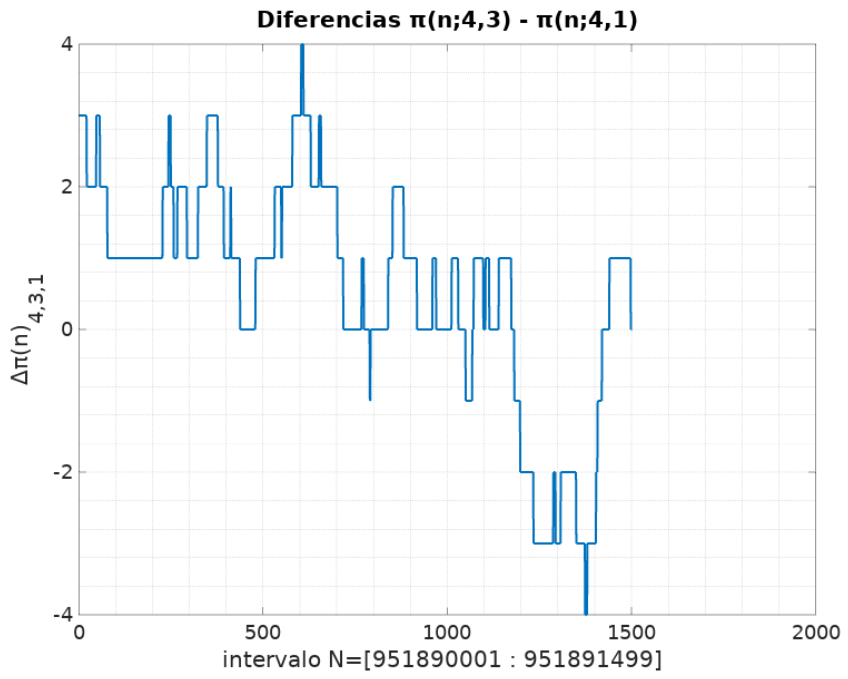

(b) Ampliación en el rango del círculo rojo de (a).

Figura 2: Búsqueda de valores negativos y cero para $\Delta \pi(n)_{4,3,1}$. (Elaboración propia).

Otros resultados interesantes, para este rango de $n \approx 10^{9}$, son:

- Valor mínimo positivo $=1$, ocurre 512 veces. Valor máximo positivo $=4$, ocurre 6 veces.

- Valor mínimo negativo $=-4$, ocurre 6 veces. Valor máximo negativo $=-1$, ocurre 48 veces.

- Valor $=0$, ocurre 328 veces.

Además, para cerrar esta sección, se muestran en la Tabla 5 los diferentes intervalos de $n$ para los dos casos de interés, es decir, donde $\Delta \pi(n)_{4,3,1}$ toma valores cero y negativos.

Tabla 5: Rangos de $n$ para valores de $\Delta \pi(n)_{4,3,1}$ cero y negativos.

\begin{tabular}{cc}
\hline RANGOS de $n$ donde $\Delta \pi(n)_{4,3,1}=0$ & RANGOS de $n$ donde $\Delta \pi(n)_{4,3,1}<0$ \\
\hline$[951890437: 951890477]$ & {$[951890789: 951890789]$} \\
{$[951890717: 951890765]$} & {$[951891049: 951891065]$} \\
{$[951890773: 951890787]$} & {$[951891181: 951891417]$} \\
{$[951890791: 951890837]$} & \\
{$[951890917: 951890957]$} & \\
{$[951890969: 951891009]$} & \\
{$[951891029: 951891047]$} & \\
{$[951891067: 951891069]$} & \\
{$[951891097: 951891101]$} & \\
{$[951891113: 951891137]$} & \\
{$[951891173: 951891179]$} & \\
{$[951891419: 951891437]$} & \\
{$[951891497: 951891501]$} & \\
\hline
\end{tabular}

Una conclusión es que, apoyados en los Teoremas 1 y 2, queda abierta la posibilidad de que el número de veces en que $\Delta \pi(n)_{4,3,1}=0$ sea infinito, es decir, que se cumpla la Conjetura 2 . Finalmente, se puede hacer una conjetura más: 


\section{Conjetura 3 (Este trabajo)}

Si designamos como $\mathrm{I}^{+}$al infinito número de veces en que $\Delta \pi(n)_{4,3,1}>0, \mathrm{I}^{-}$al infinito número de veces en que $\Delta \pi(n)_{4,3,1}<0$, e $I^{0}$ al infinito número de veces en que $\Delta \pi(n)_{4,3,1}=0$, para $n \rightarrow \infty$, tenemos que

$$
\mathrm{I}^{+}>\mathrm{I}^{0}>\mathrm{I}^{-}
$$

es decir, hay más infinitas veces en que la diferencia es mayor que cero, seguida del número de infinitas veces que es igual a cero y finalmente el número de infinitas de veces que es menor que cero.

\section{La carrera de series divergentes}

Partiendo de la idea de la carrera de los números primos y de [7] con series divergentes, se muestra a través de sumas parciales, que dos series divergentes subarmónicas-p, también tienen un comportamiento igual en su carrera al infinito, es decir, un infinito es menor que otro.

\section{Definición 4}

Una serie subarmónica-p se define como

$$
S_{p}^{\prime}=\sum_{n=1}^{\infty} \sum_{j=n^{p}+1}^{(n+1)^{p}-1} \frac{1}{j}, \text { con } p>1
$$

que forma parte de la serie armónica ${ }^{a}$ a la cual se eliminan los términos de una serie-p.

\footnotetext{
${ }^{a}$ Para ver el desarrollo completo consultar [7].
}

Una serie-p está definida como

$$
S_{p}=\sum_{n=1}^{\infty} \frac{1}{n^{p}}, \text { con } p>1 .
$$

Puede observarse que con la serie $S_{p}$ y la serie $S_{p}^{\prime}$ se forma la serie armónica, es decir, la reconstrucción de la serie armónica $S$ queda como

$$
S=\sum_{n=1}^{\infty} \frac{1}{n}=S_{p}+S_{p}^{\prime}=\sum_{n=1}^{\infty}\left(\frac{1}{n^{p}}+\sum_{j=n^{p}+1}^{(n+1)^{p}-1} \frac{1}{j}\right)
$$

Anteriormente se mostró que la (5) es divergente [7], mientras que la (6) se sabe que es convergente para $p>1$.

Para el desarrollo de estas series, conviene seguir el enfoque de los números armónicos, es decir, si la suma parcial de la serie armónica es conocida como número armónico, denotado por $\mathrm{H}_{n}$, el cual se expresa por

$$
\mathrm{H}_{\mathrm{n}}=\sum_{i=1}^{\mathrm{n}} \frac{1}{\mathrm{i}}
$$


entonces llamaremos número subarmónico-p a la suma parcial de la serie $S_{p}^{\prime}$, y lo denotaremos por $\mathrm{H}_{\mathrm{p}, \mathrm{n}}^{\prime}$

$$
H_{p, n}^{\prime}=\sum_{i=1}^{n} \sum_{j=i^{p}+1}^{(i+1)^{p}-1} \frac{1}{j} .
$$

El último término que falta definir es la suma parcial de la serie-p, con $p>1$, la cual se denotará por

$$
S_{p, n}=\sum_{i=1}^{n} \frac{1}{\mathfrak{i p}^{p}}
$$

Se conocen valores de convergencia para $p=2,4,6$ entre otros. Para valores impares de $p$ los valores de convergencia no son tan conocidos, pero la constante de Apéry, para $p=3$ es bien conocida [1]. Puede notarse en la Figura 3, que con muy pocos términos, considerando que se trata de una suma infinita, la serie se acerca al valor de convergencia. De hecho con los primeros mil términos el error entre la suma y $\pi^{2} / 6$ es de $9.9 \times 10^{-4}$ y con apenas un millón de términos el error es de $1 \times 10^{-6}$.

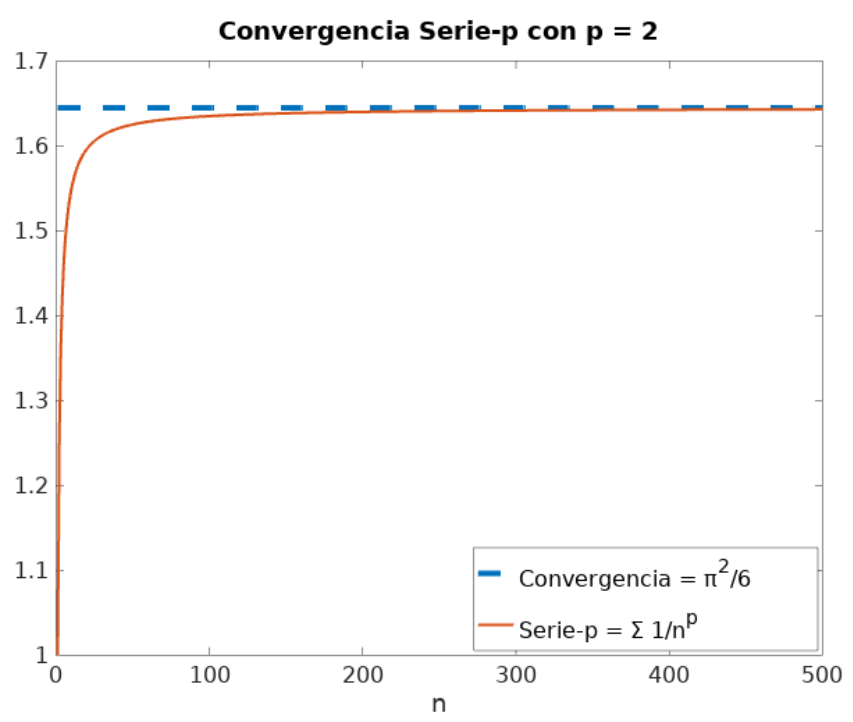

Figura 3: Convergencia hacia $\pi^{2} / 6$ de la serie-p con $p=2$. (Elaboración propia).

Dicho lo anterior se podrá ver que el número armónico $H_{n}$ se puede expresar como

$$
H_{n}=\sum_{i=1}^{n} \frac{1}{i}=S_{p, n}+H_{p, n}^{\prime}=\sum_{i=1}^{n}\left(\frac{1}{i^{p}}+\sum_{j=i^{p}+1}^{(i+1)^{p}-1} \frac{1}{j}\right) .
$$

Para verificar la (7) en la Figura 4 se muestra un caso, con $p=3$, de la construcción del número armónico $H_{n}$ como la suma del número subarmónico- $p\left(H_{p, n}^{\prime}\right)$ y su serie-p parcial $\left(S_{p, n}\right)$ asociada. Sobre la línea en color azul se puede ver, sobrepuesta, la suma $S_{p, n}+H_{p, n}^{\prime}$ la cual es aproximadamente igual al número armónico $\mathrm{H}_{n}$.

Como se mostró analíticamente en [7], la diferencia entre dos series subarmónicas-p divergentes es igual a la diferencia entre sus series-p convergentes asociadas. Empleando ahora las sumas parciales, podemos expresar

$$
H_{n}=H_{p_{1}, n}^{\prime}+S_{p_{1}, n}=H_{p_{2}, n}^{\prime}+S_{p_{2}, n}
$$

de modo que

$$
H_{p_{1}, n}^{\prime}-H_{p_{2}, n}^{\prime}=S_{p_{2}, n}-S_{p_{1}, n}
$$




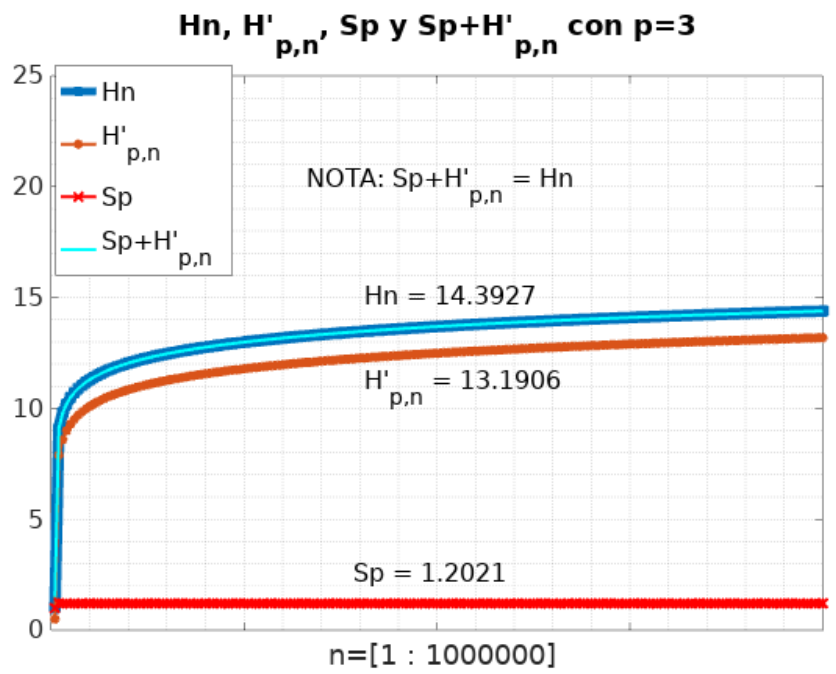

Figura 4: $H_{n}$ como suma de $H_{p, n}^{\prime}+S_{p, n}$ con $p=3$. (Elaboración propia).

es decir, la diferencia entre el número subarmónico- $p_{1}$ y el número subarmónico- $p_{2}$ es igual a la diferencia entre su series-p parciales asociadas. Al tender $n$ al infinito se puede ver este desarrollo como una carrera entre dos series divergentes.

Una forma de verificar esta carrera se muestra en la Figura 5. En la misma figura se muestra la diferencia entre los números subarmónicos, la diferencia entre las series parciales $S_{p, n}$ y la diferencia entre los valores de convergencia ya conocidos al infinito.

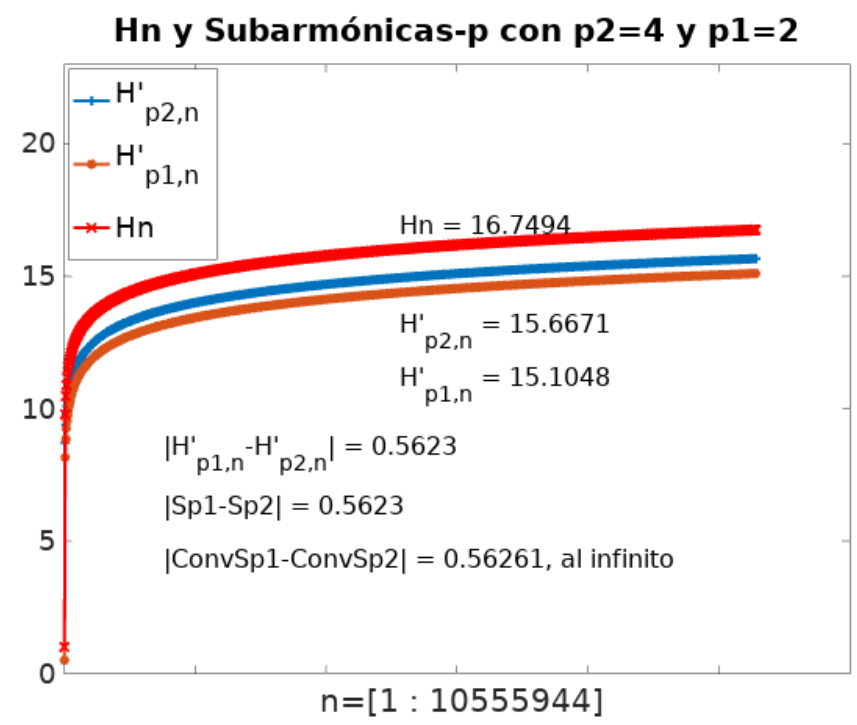

Figura 5: Números subarmónicos-p: $\mathrm{H}_{2, n}$ y $\mathrm{H}_{4, n}$. (Elaboración propia).

Se espera que al aumentar el número de términos, en la carrera, la diferencia entre las series subarmónicas-p será igual a la diferencia de las series-p, como se puede observar en la Tabla 6 para diferentes valores de $n$ y diferentes valores de $p$. La carrera se puede realizar para cualesquiera par de valores $p_{1}$ y $p_{2}$. Para conocer el valor de convergencia asintótico de las series-p, se utilizó la función gsl_sf_zeta_int en Octave, la cual calcula la función zeta de Riemann para $n$ entero $n \neq 1$. En los cálculos se emplea la suma parcial de la serie-p la cual fue denotada por $S_{p, n}$, donde $n$ será el valor equivalente para el número armónico correspondiente. En todos los casos, si extendemos n al infinito, la diferencia entre los números subarmónicos (que se convierte en la diferencia entre series subarmónicas) será igual a la diferencia entre los valores de convergencia al infintio entre $S_{p_{1}}$ y $S_{p_{2}}$, tal y como se demostró en [7]. Debe tomarse en cuenta que al desarrollar los números armónicos 
Tabla 6: Carrera entre números subarmónicos-p y su valor asintótico: 0.442877163688632 parte superior, 0.562610833137088 parte inferior.

\begin{tabular}{ccccc}
\hline $\mathrm{n}$ & $\mathrm{p}_{1}, \mathrm{p}_{2}$ & $\mathrm{H}_{\mathrm{p}_{1}, \mathrm{n}}^{\prime}-\mathrm{H}_{\mathrm{p}_{2}, \mathrm{n}}^{\prime}$ & $\mathrm{S}_{\mathrm{p}_{2}}-\mathrm{S}_{\mathrm{p}_{1}}$ & Error \\
\hline 10626 & 2,3 & 0.437871781251198 & 0.434202225009704 & 0.00366955624149456 \\
103776 & 2,3 & 0.441337680664201 & 0.439997960166588 & 0.00133972049761311 \\
999900 & 2,3 & 0.441927166021866 & 0.441927166021883 & $1.70974345792274 \mathrm{e}-14$ \\
10077480 & 2,3 & 0.442282320302468 & 0.442282320302470 & $2.66453525910038 \mathrm{e}-15$ \\
\hline 104958 & 2,4 & 0.559581740541230 & 0.559581740541209 & $2.04281036531029 \mathrm{e}-14$ \\
1048544 & 2,4 & 0.561644452937180 & 0.561644452937174 & $6.66133814775094 \mathrm{e}-15$ \\
10555944 & 2,4 & 0.562304846598773 & 0.562304846598774 & $1.55431223447522 \mathrm{e}-15$ \\
99999900 & 2,4 & 0.562511166500954 & 0.562511166500953 & $1.33226762955019 \mathrm{e}-15$ \\
\hline
\end{tabular}

con la (7), aunque el valor de $n$ final de cada número armónico es cercano a $10^{8}$ para el número más grande, los números de las series-p parciales son mucho menores, ya que se debe igualar el número de términos en cada serie para que la comparación (8) sea válida. Por ejemplo, en la construcción de la Figura 5 se tiene $n_{2}=56$, y $n_{1}=3248$ y de acuerdo a la (7) los números armónicos se extienden hasta $n=10555944$. Por esa razón en la Tabla 6 los valores de la cuarta columna están aún alejados de su valor asintótico al infinito. Sin embargo, se mantiene muy cercano al valor de la tercera columna, tal y como lo predice la (8).

\section{Conclusiones}

En este trabajo se presentó una observación sobre la carrera de números primos, remarcando la importancia de tomar en cuenta el número de veces en que la carrera se empata. Se exhibieron ejemplos

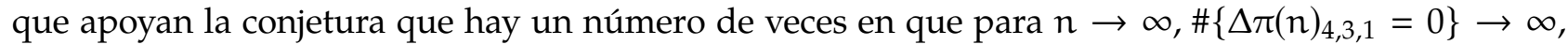
es decir, se conjetura que el número de veces en que el número de primos de la forma $4 n+3$ es igual al número de primos de la forma $4 n+1$, es infinito. Bajo la observación de una cantidad finita de casos, se encontró que esto ocurre menos veces que la observación de Chebyshev, y más veces que la distribución de Littlewood, aunque todas ocurran un número infinito de veces, un infinito es menor que el otro.

La revisión de una distribución de números primos, se usó como marco de referencia, para posteriormente desarrollar series subarmónicas parciales divergentes, así como las sumas parciales de las series-p convergentes. Una vez calculados estos números que llamamos subarmónicos-p, se mostró que mantienen una diferencia con un valor asintótico a la diferencia de sus series-p correspondientes. De este modo, se puede decir que en la carrera hacia el infinito entre dos series subarmónicas-p divergentes, una va detrás de la otra con un valor que se puede calcular con la (8), como se muestra en la Tabla 6.

\section{Bibliografía}

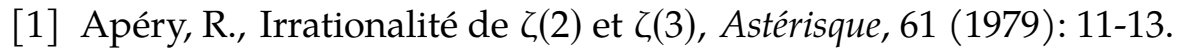

[2] Eaton, J. W., Bateman, D., Hauberg, S., and Wehbring, R., Octave version 4.2.1 manual: a highlevel interactive language for numerical computations. https://wWw. gnu . org/sof twa re/octave/ doc/v4.2.1/. 
[3] Chebyshev, P.L., Lettre de M. le Professeur Tchébychev à M. Fuss sur un nouveaux théorème relatif aux nombres premiers contenus dans les formes $4 n+1$ et $4 n+3$, Bull. Classe Phys. Acad. Imp. Sci. St. Petersburg, 11 (1853), 208.

[4] Granville, A., \& Martin, G., (2006). Prime Number Races. The American Mathematical Monthly, 113(1), 1-33. doi:10.2307/27641834

[5] Knapowski, S., and Turán, P., Comparative prime-number theory, I-III, Acta Math. Acad. Sci. Hungar. 13 (1962) 299-364.

[6] Littlewood, J. E., Distribution des nombres premiers, C. R. Acad. Sci. Paris 158 (1914) 1869-1872.

[7] Miramontes-de León, D., y Miramontes-de León, G., Los infinitos de algunas series divergentes. Revista Digital Matemática, Educación e Internet, 20(2), 2020. Recuperado de: https://tecdigital.tec.ac.cr/revistamatematica/ArticulosRevistaDigitalV20_n2 2020/RevistaDigital_Miramontes_V20_n2_2020/

[8] Rubinstein, M., and Sarnak, P., Chebyshev's bias, Experiment. Math. 3 (1994) 173-197. 